\title{
OPEN Catalytic oxidation of small organic molecules by cold plasma in solution in the presence of molecular iron complexes ${ }^{\dagger}$
}

\author{
Dariusz Śmiłowicz ${ }^{1}$, Friederike Kogelheide ${ }^{2}$, Anna Lena Schöne ${ }^{2}$, Katharina Stapelmann ${ }^{3}$, \\ Peter Awakowicz ${ }^{2}$ \& Nils Metzler-Nolte ${ }^{1 \bowtie}$
}

The plasma-mediated decomposition of volatile organic compounds has previously been investigated in the gas phase with metal oxides as heterogeneous catalysts. While the reactive species in plasma itself are well investigated, very little is known about the influence of metal catalysts in solution. Here, we present initial investigations on the time-dependent plasma-supported oxidation of benzyl alcohol, benzaldehyde and phenol in the presence of molecular iron complexes in solution. Products were identified by HPLC, ESI-MS, FT-IR, and ${ }^{1}$ H NMR spectroscopy. Compared to metal-free oxidation of the substrates, which is caused by reactive oxygen species and leads to a mixture of products, the metal-mediated reactions lead to one product cleanly, and faster than in the metal-free reactions. Most noteworthy, even catalytic amounts of metal complexes induce these clean transformations. The findings described here bear important implications for plasma-supported industrial waste transformations, as well as for plasma-mediated applications in biomedicine, given the fact that iron is the most abundant redox-active metal in the human body.

The chemical and pharmaceutical industries produce a large amount of wastes and pollutants ${ }^{1-3}$. The selective removal or conversion of air and water pollutants consisting of volatile organic compounds (VOCs) constitutes one of the biggest challenge in modern manufacturing ${ }^{4-6}$. Recently, treatment by non-thermal dielectric-barrier discharge (DBD) plasma has received some attention in waste management to provide selective oxidation of hazardous, organic compounds in the chemical industry ${ }^{7-9}$. Since DBD plasma generates vast numbers of reactive species, such as reactive nitrogen and oxygen species, ozone and hydrogen peroxide, it has been utilized to convert toxic compounds to non-hazardous oxidation products ${ }^{10-12}$. So far, heterogeneous catalysis has been involved to neutralize volatile organic compounds ${ }^{13-15}$. Small organic molecules like phenol, benzene, toluene, butyraldehyde and triclocarban were treated with DBD plasma in the presence of metal-oxides as catalysts, including $\mathrm{TiO}_{2}, \mathrm{MnO}_{2}, \mathrm{Fe}_{2} \mathrm{O}_{3}$ and $\mathrm{CeO}_{2}{ }^{16-18}$. Unfortunately, this resulted in low percentage of conversion only, and numerous oxidation by-products ${ }^{19,20}$. Recently, we investigated the time-dependent chemical modifications of glutathione (GSH) and oxidized glutathione dimer (GSSG) caused by DBD plasma under ambient, aqueous conditions in the presence and absence of iron(II) and iron(III) complexes. We showed that involving either iron(II) or iron(III) complexes in stoichiometric amounts resulted in one clean main oxidation product, namely glutathione sulfonic acid $\left(\mathrm{GSO}_{3} \mathrm{H}\right)^{21}$. These results are in contrast to results gained when GSH / GSSG was treated with a DBD plasma alone, which gave a broad mixture of products ${ }^{22,23}$. Encouraged by those previous results, we decided to explore the plasma-assisted oxidation of small molecules under ambient conditions in solution, and in the presence of the same stable iron compounds as homogeneous catalysts, namely ferrocenecarboxylic acid (A) and hemin (B, Fig. 1). In exploratory work we describe in this communication the treatment of organic model compounds (benzyl alcohol, benzaldehyde and phenol, Fig. 1) in aqueous solutions containing iron complexes $\mathbf{A}$ and $\mathbf{B}$ with a dielectric barrier discharge (DBD) plasma.

DBDs are known to generate a wide range of reactive species, such as ozone or nitric oxide ${ }^{24-26}$, depending on the exact conditions ${ }^{27,28}$. A standardized DBD was used in this work, which was already extensively characterised by us in previous studies regarding its plasma parameters, such as the electron density or the gas temperature,

${ }^{1}$ Inorganic Chemistry I - Bioinorganic Chemistry, Ruhr University Bochum, 44780 Bochum, Germany. ${ }^{2}$ Institute for Electrical Engineering and Plasma Technology, Ruhr University Bochum, 44780 Bochum, Germany. ${ }^{3}$ Department of Nuclear Engineering, North Carolina State University, Raleigh, NC 27695, USA. ${ }^{\square}$ email: nils.metzler-nolte@ rub.de 
a)<smiles>OCc1ccccc1</smiles><smiles>O=Cc1ccccc1</smiles><smiles>Oc1ccccc1</smiles>

b)

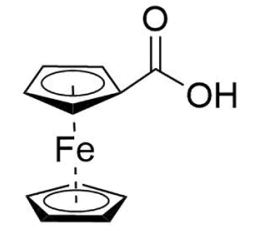

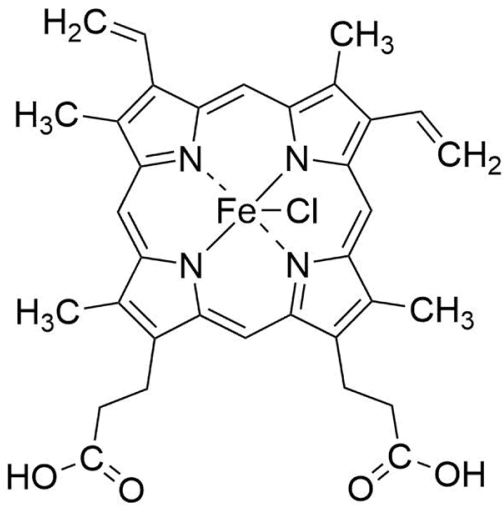

B

Figure 1. Structures of (a) substrates and (b) iron complexes.

and the generated concentration of reactive chemical species ${ }^{29,30}$. Among others, the ozone density amounts to $7 \times 10^{16} \mathrm{~cm}^{-3}$ and the measured NO density is equal to $1.2 \times 10^{15} \mathrm{~cm}^{-3}$ for the operation parameters used in this study ${ }^{30}$. Moreover, optical emission spectroscopy (OES) was applied to measure the UV irradiance emitted by the DBD. The UV-A and UV-B irradiance amounts to $1.1 \times 10^{-4} \mathrm{~J} \mathrm{~s}^{-1} \mathrm{~cm}^{-2}$ and $1.1 \times 10^{-5} \mathrm{~J} \mathrm{~s}^{-1} \mathrm{~cm}^{-2}$, respectively ${ }^{30}$. The present studies were carried out under the same experimental conditions, namely under ambient air, at room temperature and at atmospheric pressure.

\section{Results}

In a first step, the time-dependent impact of DBD treatment on the organic molecules alone, i.e. without iron complexes was investigated. Benzyl alcohol (compound 1), benzaldehyde (compound 2) and phenol (compound 3) were treated with plasma for 1, 3, 5 and $20 \mathrm{~min}$ in aqueous solution. Treatment of 1 with the DBD for 1 min revealed a mixture of products according the HPLC trace of the solution. Extending the treatment time to $3,5 \mathrm{~min}$ as well as to $20 \mathrm{~min}$ caused no further changes in the distribution of products (Fig. 2a).

Among the mixture of products, we were able to identify four compounds by mass spectrometry (signals in the ESI-MS at $m / z=44,86,107$ and 123). All signals correspond to $[\mathrm{M}+\mathrm{H}]^{+}$species (Fig. $3 \mathrm{a}$ ) and they were assigned to propene, hexa-2,4-dien, benzaldehyde and benzoic acid, respectively (Fig. 5a). Compound 2, when treated with plasma, displayed the same products after 1,3,5 and $20 \mathrm{~min}$. When compound 3 was treated with plasma alone, the resulting HPLC and ESI-MS data showed a mixture of products (phenol, biphenyl, 2,2'-biphenol, benzoic acid), mostly the same as those observed for benzene oxidation using DBD by Dey et al. ${ }^{31}$.

In the next step, the impact of plasma on compounds 1, $\mathbf{2}$ and $\mathbf{3}$ in the presence of a stoichiometric amount of ferrocenecarboxylic acid (complex A) and hemin (complex B) was investigated. Both iron complexes were chosen for this work because they exhibit high stability in aqueous medium during plasma treatment, as shown in a previous study ${ }^{21}$. They differ however in the oxidation state of iron, with $\mathbf{A}$ being $\mathrm{Fe}(+\mathrm{II})$, and the Fe center in hemin (B) being +III. For benzyl alcohol after 1 and $3 \mathrm{~min}$ of plasma treatment in the presence of iron(III) complex B only two compounds were detected, namely benzaldehyde and benzoic acid (Fig. 5b). However, after $5 \mathrm{~min}$, benzoic acid was observed as the only species, indicating a complete oxidation of the benzylic group, whereas without iron complex, benzoic acid constitutes only $8 \%$ of all final products (Fig. 2a). Extending the plasma treatment time to $20 \mathrm{~min}$ did not influence the number or structure of products. Also, treatment of $\mathbf{1}$ with plasma in the presence of $\mathbf{A}$ resulted largely in the same products. The data are available in the ESI $\dagger$. The HPLC profile after $20 \mathrm{~min}$ of plasma treatment in the presence of both iron complexes showed one signal at $7.2 \mathrm{~min}$ (cf. Fig. $2 b$ ) with $m / z$ value at 123 on ESI-MS spectrum (Fig. $3 b$ ). The same oxidation pattern was observed in the case of benzaldehyde. After 1 min of plasma treatment with complexes A or $\mathbf{B}$ two compounds were observed according to HPLC and ESI-MS analysis, namely benzaldehyde and benzoic acid. After 3, 5 and 20 min only 


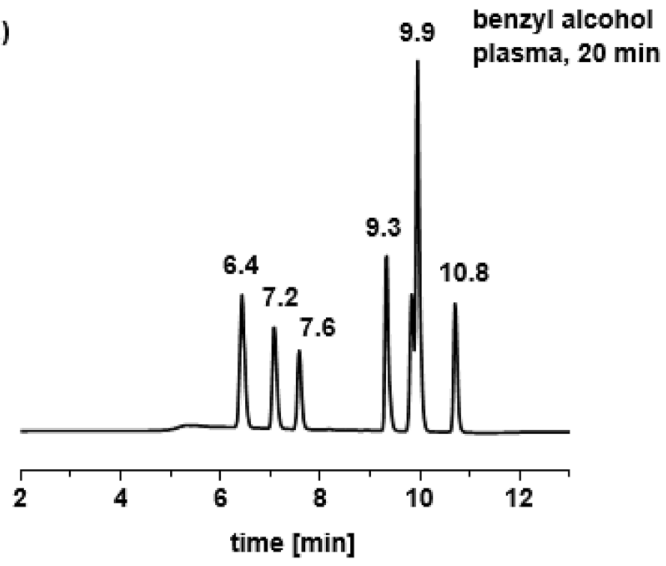

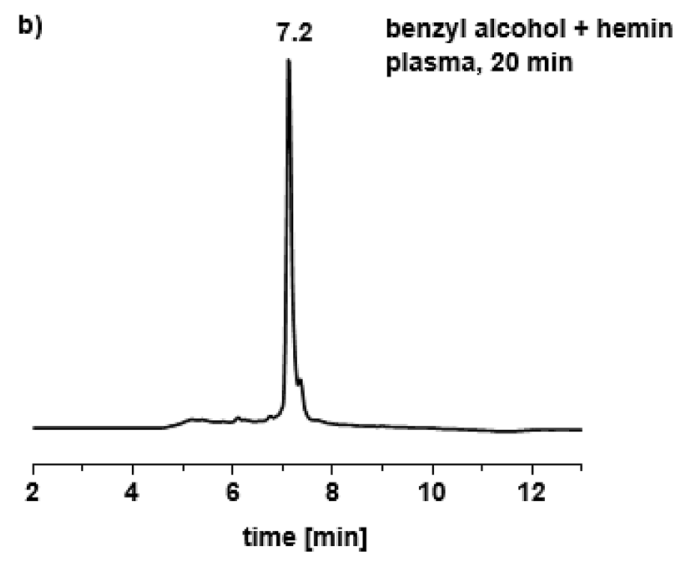

Figure 2. HPLC trace of benzyl alcohol after plasma treatment (a) without metal complex, (b) in the presence of complex $\mathbf{B}$.

a)

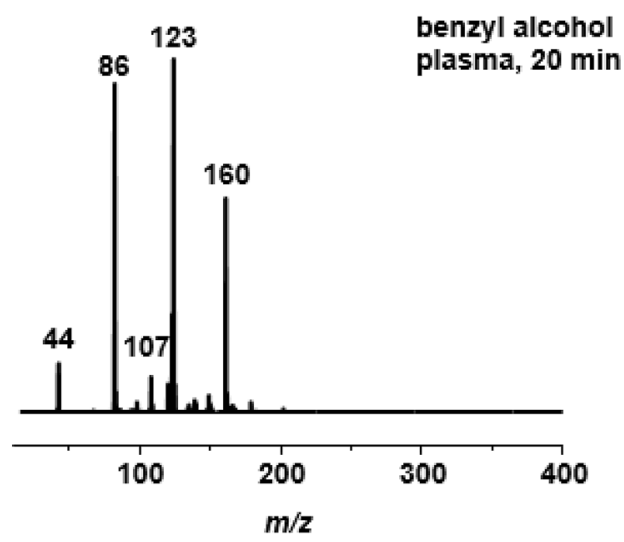

b)

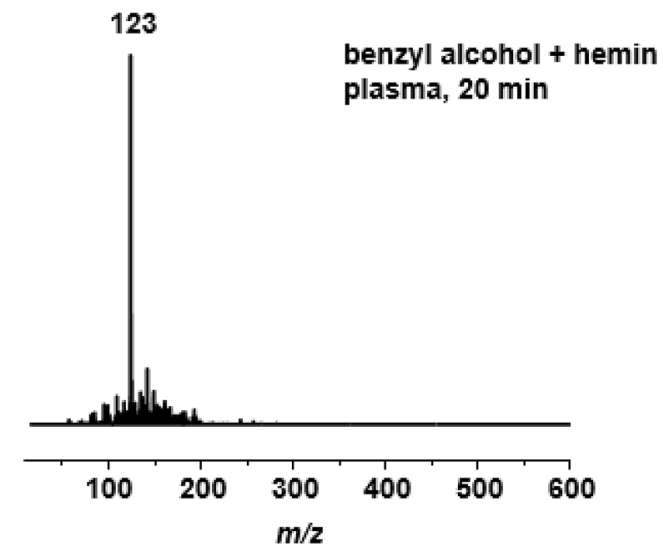

Figure 3. ESI-MS spectrum of benzyl alcohol after plasma treatment (a) without metal complex, (b) in the presence of complex B.

benzoic acid was observed as the sole oxidation product. However, in the case of phenol, several products with functional groups were observed (benzaldehyde, benzoic acid), among many products after 20 min of plasma treatment $(\mathrm{ESI} \dagger)$.

FT-IR spectroscopy confirmed the presence of the carboxylic acid group after plasma treatment with a peak at $1685 \mathrm{~cm}^{-1}$ for the $\mathrm{C}=\mathrm{O}$ stretching vibration $(\mathrm{ESI} \dagger)$, which agrees with the characteristic bands for benzoic acid in literature ${ }^{32} .{ }^{1} \mathrm{H}$ NMR spectroscopy ultimately confirmed nature of the product after oxidation of substrates by DBD plasma treatment (Fig. 4) by showing the protons from the aromatic ring at 7.45-7.96 ppm and a signal at $12.88 \mathrm{ppm}$ corresponding to the $\mathrm{OH}$ from the carboxylic acid group of benzoic acid. Notably, the absence of an aldehyde proton around $10 \mathrm{ppm}$ indicates complete conversion with no intermediate oxidation product left. Also notably, the clean ${ }^{1} \mathrm{H}$ NMR spectrum excludes any further oxidation or degradation of benzoic acid.

In order to establish whether these rather clean conversions were stoichiometric in nature the DBD-supported oxidation was carried out in the presence of $10 \%$ of the iron complexes. For all substrates the oxidation pattern appeared to be identical, with benzoic acid as main oxidation product, in comparison to experiments carried out with the stoichiometric amount (ESI $\dagger$ ). According to the results (cf. Fig. 5), the oxidation products do not depend on the nature of iron complexes, i.e. the $\mathrm{Fe}(\mathrm{II})$ and $\mathrm{Fe}$ (III) complexes give an identical spectrum of products, regardless of the oxidation state of the Fe center. Only small time-dependent differences can be observed since the oxidation to the final product is accomplished faster in the presence of hemin, the iron(III) complex.

To complete the picture, two sets of control experiments were carried out: Stability experiments in distilled water for molecules 1, 2 and 3 alone, and interaction studies of 1, 2 and 3 with both iron(II) and iron(III) complexes, all investigated by HPLC and ESI-MS. All organic substrates were completely stable after 1, 3, 5 and $20 \mathrm{~min}$, making them suitable candidates for plasma investigations. The HPLC traces and the full-scan mass spectra are available in the ESI $\dagger$. The iron complexes were incubated with compounds $\mathbf{1}, \mathbf{2}$ and 3 in aqueous solution for 1, 3, 5 and $20 \mathrm{~min}$ in the ratio $1: 1$, as well as with the substoichiometric amount (10\%) of iron complexes. During the whole incubation time no modification of the organic molecules nor of the iron complexes was observed. The stability of the iron complexes in aqueous solution as well as the impact of plasma on those complexes was investigated by us previously ${ }^{21}$. Both iron(II) and iron(III) complexes exhibited high stability 
a) benzyl alcohol b) benzyl alcohol + hemin
plasma, $20 \mathrm{~min}$

$\begin{array}{rrrrrrrrrrrrrrr}14 & 13 & 12 & 11 & 10 & 9 & 8 & 7 & 6 & 5 & 4 & 3 & 2 & 1 & 0\end{array}$

Figure 4. ${ }^{1} \mathrm{H}$ NMR spectrum of (a) benzyl alcohol, (b) benzyl alcohol after $20 \mathrm{~min}$ of plasma treatment in the presence of complex $\mathbf{B}$ (both spectra recorded in DMSO-d6).

in aqueous medium during plasma treatment with no traces of decomposition, which makes them suitable candidates for the above studies.

Since both iron complexes possess pendant carboxylate groups, additional control experiments were carried out using benzoic acid as the catalyst to prove that the iron centre and not a carboxylate unit per se is responsible for the observed oxidation results. Benzyl alcohol, benzaldehyde and phenol were treated by cold plasma in the presence of a stoichiometric amount of benzoic acid for 1, 3 and 5 min. HPLC and ESI-MS analyses revealed oxidation patterns similar to those obtained from the experiments without iron (II) and iron (III) complexes. At the same time, benzoic acid appeared to be stable during plasma treatment in the absence of metal complexes (ESI $\dagger$ ).

\section{Discussion}

In summary, we report here the use of iron complexes as catalysts for the specific transformation of organic substrates under the influence of cold, atmospheric pressure plasma. Degradation as well as a whole range of oxidation products were observed for compounds 1, 2 and 3 after DBD plasma treatment alone, while only one oxidation product was more rapidly found after plasma treatment in the presence of stoichiometric as well as substoichiometric (10\%) amounts of the iron complexes A and B. Chromatography as well as spectroscopic analysis reveal that benzoic acid is the main oxidation product after Fe-assisted plasma treatment of benzyl alcohol and benzaldehyde under ambient air, at room temperature and at atmospheric pressure. Ferrocenecarboxylic acid (with $\mathrm{Fe}$ (II)) as well as the Fe(III) hemin complex are suitable for homogeneous oxidation of these organic compounds by DBD plasma, since both species are redox active. Details of how these metal complexes interact with the reactive species initially generated by the plasma remain elusive at these early stages of investigation, but general chemical wisdom would suggest Fenton-type and / or dismutase-like chemistry involving redox cycling between $\mathrm{Fe}(\mathrm{II})$ and $\mathrm{Fe}$ (III) species. This would also explain why compounds $\mathbf{A}$ and $\mathbf{B}$ are more or less equally active in our experiments, irrespective of the different oxidation states of the iron complexes originally. What is crucial however is to use stable iron complexes, because as we reported previously, some iron(III) complexes, for example iron(III) acetylacetonate undergo decomposition during plasma treatment and further interact with substrates during plasma treatment ${ }^{21}$.

The findings of this investigation complement those of earlier studies involving DBD plasma for oxidation assistance. Nevertheless, two important new aspects emerge from our investigations above. First, metal complexes - as exemplified by redox-active iron compounds in this study - are able to work in concert with plasma-generated species, like ROS, and they do so even at catalytic amounts. The metal complexes will not necessarily make the oxidation reaction faster, but can drive the reaction towards a more uniform product spectrum. This may be 
a)

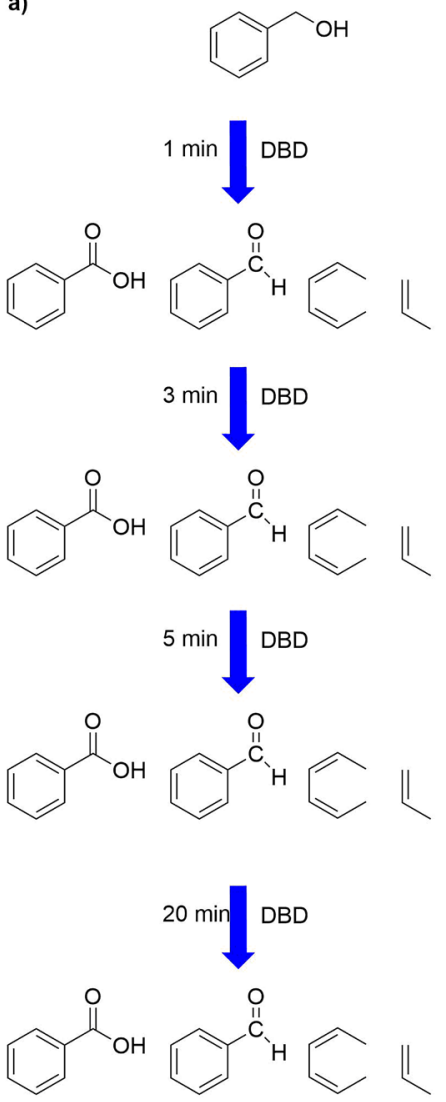

b)

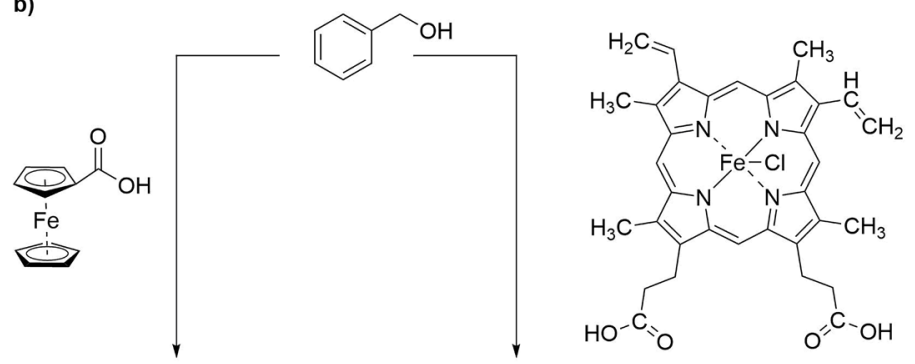

$1 \min \mathrm{DBD}$
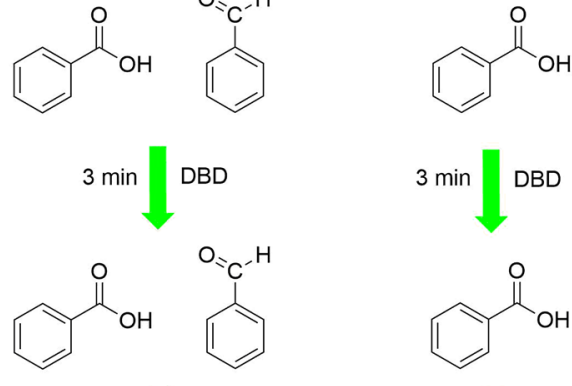

$\overbrace{\mathrm{OH}}^{\mathrm{O}}$

5 min DBD<smiles>O=C(O)c1ccccc1</smiles>

$20 \mathrm{~min}$ DBD<smiles>O=C(O)c1ccccc1</smiles>

Figure 5. Chemical modifications of benzyl alcohol after different times of plasma treatment (a) without iron complexes, $(\mathbf{b})$ in the presence of iron complexes.

highly desirable for industrial applications, such as transformation of organic waste products from an industrial process to one, possible value-added product rather than a product mixture that must be considered waste.

The second important conclusion from this work concerns the application of cold plasma in biology and medicine, e.g. in wound healing ${ }^{33,34}$. So far, reactive species like ROS and RNS were considered responsible for triggering the biological responses. However, metals are almost ubiquitous in biology as cofactors in metalloenzymes, and iron in particular is by far the most abundant redox-active metal in the human body, prominently present in hemoglobin. It is highly likely that metal-containing biomolecules, like hemoglobin, play an important role in mediating the presence and nature of plasma-generated species, and will thereby have a decisive influence on the biological outcome of plasma-triggered medical treatments. This aspect has not been considered in previous work at all. It will be the focus of future investigations from our groups.

\section{Materials and methods}

Experimental setup. The experimental setup used in this work has been described in a similar way before $^{21,22}$. Briefly, the experiments in this study were carried out with a dielectric barrier discharge, which consists of a copper electrode covered with aluminium oxide $\left(\mathrm{Al}_{2} \mathrm{O}_{3}\right)$ with a thickness of $1 \mathrm{~mm}$. The electrode has a diameter of $10 \mathrm{~mm}$ and the distance between the driven electrode and the sample was kept constant at $1 \mathrm{~mm}$. The samples were placed on a grounded aluminium plate and ambient air was used as the process gas. The temperature in the lab was adjusted to $20^{\circ} \mathrm{C}$ and the relative humidity varied between 40 and $50 \%$ during the period in which the experiments were carried out. The experimental setup is described in more detail in Kogelheide et al. and a scheme of the dielectric barrier discharge can be found in the ESI, Scheme $\mathrm{S}^{\dagger 35}$. The electrode was driven with a pulsed power supply ${ }^{29}$. For the experiments in this study the repetition frequency was set to $300 \mathrm{~Hz}$ and the amplitude of the $\mathrm{HV}$ pulse to $24 \mathrm{kV}_{p p}$. The dielectric barrier discharge has been characterised regarding several plasma parameters as well as reactive species. In Kogelheide et al., the electron density distribution in the discharge is described in detail ${ }^{29}$. The radial profiles of the plasma produced oxygen species, atomic oxygen $(\mathrm{O})$ and ozone $\left(\mathrm{O}_{3}\right)$, within the plasma volume of the former used plasma source are determined using two-photon 
laser-induced fluorescence spectroscopy (TALIF) and optical absorption spectroscopy (OAS) in Baldus et al. Furthermore, a model of the afterglow chemistry is described in this paper to obtain insight into the dynamics of the considered reactive oxygen species ${ }^{36}$.

Materials. All reagents and chemicals were purchased from commercial sources and used without further purification. Ferrocenecarboxylic acid was purchased from Abcr. Chloro(protoporphyrinato)iron(III) (hemin), benzyl alcohol, benzaldehyde, phenol and N,N-diisopropylethylamine (DIEA) were purchased from SigmaAldrich.

Samples preparation. A similar protocol for sample treatment was published by us previously ${ }^{21}$. Small organic molecules were dissolved in distilled water with a concentration of $4 \mathrm{mg} / \mathrm{ml}$. Ferrocenecarboxylic acid and chloro(protoporphyrinato)iron(III) (hemin) were dissolved in distilled water with a concentration of $4 \mathrm{mg} /$ $\mathrm{ml}$ with 2 eq. of DIEA. $10 \mu \mathrm{l}$ were placed on cleaned silicon wafers (Siltronic AG) and treated with the DBD for $1,3,5$ and $20 \mathrm{~min}$. After treatment, samples were filled into reagent tubes and evaporated liquid replenished with distilled water to the concentration of $1 \mathrm{mg} / \mathrm{mL}$ for the analysis via mass spectrometry and HPLC. The samples for the FTIR spectroscopy were dried by desiccation after the plasma treatment. As controls, another sample was prepared equally, omitting the plasma treatment. Control samples were placed in ambient conditions as the sample treated for the longest time.

Mass spectrometry. Electron spray ionization (ESI) mass spectra were obtained on an Esquire 6000 mass spectrometer (Bruker). Analysis of samples by ESI-MS follows our standard operating procedure that has been described before ${ }^{21,22}$. Full mass spectra of the investigated ferrocenecarboxylic acid, chloro(protoporphyrinato) iron(III) (hemin), benzyl alcohol, benzaldehyde and phenol were acquired in both negative-ion and positive-ion mode with the spectrometer equipped with an ion-trap analyser. Three samples of $10 \mu l$ treated for the same time were pooled and diluted tenfold with acetonitrile for $300 \mu \mathrm{l}$ with a final concentration of $1 \mathrm{mg} / \mathrm{ml}$. Instrumental parameters were tuned for each sample. The capillary voltage was set in a range of -22 to $25 \mathrm{~V}$, the spray voltage was between 3.00 and $4.50 \mathrm{kV}$, and a capillary temperature of $180^{\circ} \mathrm{C}$ was employed. The mass scan range was from $\mathrm{m} / z 50$ to $2000 \mathrm{amu}$, for $20 \mathrm{~s}$ scan time. Spectra were acquired using a direct infusion setup with a flow rate of $5 \mu \mathrm{l} / \mathrm{min}$ with a cone voltage of $20 \mathrm{kV}$. To determine occurring in-source fragments, which increase the sample complexity without yielding significant additional information, MS/MS spectra were acquired using the same conditions with a collision energy ramp between 2.00 and $4.00 \mathrm{eV}$. Spectra were deconvoluted and a background of ten times noise (500 counts in positive and 5 counts in negative mode) was subtracted before peak annotation. All experiments were performed in triplicates.

FTIR spectroscopy. Analysis of samples by FTIR follows our standard operating procedure that has been described before ${ }^{21,22}$. A Bruker Vertex FTIR-micro spectrometer was used for the analysis of the samples. FTIR spectra were recorded from 750 to $4000 \mathrm{~cm}^{-1}$ with a spectral resolution of $4 \mathrm{~cm}^{-1}$. For the FTIR spectroscopy of the investigated ferrocenecarboxylic acid, chloro(protoporphyrinato)iron(III) (hemin), benzyl alcohol, benzaldehyde and phenol, 12 spectra were recorded at different positions of each sample with each spectrum representing 32 accumulated spectra. Background spectra were obtained for every samples due to the ambient measurement conditions to compensate water and carbon dioxide content in air ${ }^{37}$. All recorded transmission spectra, $T$, were converted into absorption spectra, $A$ :

$$
A=\log \left(\frac{1}{T}\right)
$$

Absorption spectra were baseline corrected afterwards and normalization of the data was carried out applying the Euclidean norm:

$$
a_{k}^{\text {norm }}=\frac{a_{k}}{\sqrt{\sum_{k=1}^{n}\left(a_{i}\right)^{2}}} .
$$

Every data point of each spectrum $a_{k}$ of wavenumber $k$ is normalized to the square root of the sum of every spectrum data point. All experiments were performed in triplicates.

HPLC. Analysis of samples by HPLC follows our standard operating procedure that has been described before $^{21}$. An HPLC Knauer system with a quaternary pump and a UV-DAD detector equipped with a Nucleodur $\mathrm{C} 4 \mathrm{ec}$ column $(125 \mathrm{~mm} \times 4 \mathrm{~mm}$, internal diameter $5 \mu \mathrm{m}$, Macherey-Nagel), was used. HPLC was performed by using two buffer systems (buffer $\mathrm{A}: \mathrm{H}_{2} \mathrm{O} / \mathrm{MeCN} / \mathrm{TFA}$, 95:5:0.1, v/v/v; buffer B: $\mathrm{MeCN} / \mathrm{H}_{2} \mathrm{O} / \mathrm{TFA}$, 95:5:0.1, $\mathrm{v} / \mathrm{v} / \mathrm{v})$ as the mobile phase. Chromatography was performed with a linear gradient conditions of buffer B (100\% in $10 \mathrm{~min}$ ) from $100 \%$ buffer A with a total run time of $50 \mathrm{~min}$. The flow rate of the mobile phase was $1.0 \mathrm{ml} /$ min. $10 \mu \mathrm{l}$ of the sample was injected. The column was purged with the mobile phase for $2 \mathrm{~min}$, followed by equilibration for $15 \mathrm{~min}$, and then $15 \mathrm{~min}$ were required for sample analysis. Spectral data were collected at detection wavelengths of $214 \mathrm{~nm}$ and $254 \mathrm{~nm}$, and finally the collected data were plotted (Fig. S1). 
Received: 21 August 2020; Accepted: 23 November 2020

Published online: 10 December 2020

\section{References}

1. Karuppiah, J. et al. Abatement of mixture of volatile organic compounds (vocs) in a catalytic non-thermal plasma reactor. J. Hazard. Mater. 237, 283-289. https://doi.org/10.1016/j.jhazmat.2012.08.040 (2012).

2. Raymond, M. J., Slater, C. S. \& Savelski, M. J. Lca approach to the analysis of solvent waste issues in the pharmaceutical industry. Green Chem. 12, 1826-1834. https://doi.org/10.1039/C003666H (2010).

3. Gupta, V. K., Ali, I., Saleh, T. A., Nayak, A. \& Agarwal, S. Chemical treatment technologies for waste-water recycling-an overview. Rsc Adv. 2, 6380-6388. https://doi.org/10.1039/C2RA20340E (2012).

4. Badawy, M. I., Wahaab, R. A. \& El-Kalliny, A. Fenton-biological treatment processes for the removal of some pharmaceuticals from industrial wastewater. J. Hazard. Mater. 167, 567-574. https://doi.org/10.1016/j.jhazmat.2009.01.023 (2009).

5. Van Durme, J., Dewulf, J., Leys, C. \& Van Langenhove, H. Combining non-thermal plasma with heterogeneous catalysis in waste gas treatment: a review. Appl. Catal. B 78, 324-333. https://doi.org/10.1016/j.apcatb.2007.09.035 (2008).

6. Descorme, C., Gallezot, P., Geantet, C. \& George, C. Heterogeneous catalysis: a key tool toward sustainability. ChemCatChem 4, 1897-1906. https://doi.org/10.1002/cctc.201200483 (2012).

7. Wang, L., Zhao, Y., Liu, C., Gong, W. \& Guo, H. Plasma driven ammonia decomposition on a fe-catalyst: eliminating surface nitrogen poisoning. Chem. Commun. 49, 3787-3789. https://doi.org/10.1039/C3CC41301B (2013).

8. Ye, J.-Y. \& Liu, C.-J. Cu 3 (btc) 2: Co oxidation over mof based catalysts. Chem. Commun. 47, 2167-2169. https://doi.org/10.1039/ C0CC04944A (2011).

9. Schücke, L. et al. Conversion of volatile organic compounds in a twin surface dielectric barrier discharge. Plasma Sources Science and Technology. https://doi.org/10.1088/1361-6595/abae0b (2020).

10. Kovačević, V. V. et al. Measurement of reactive species generated by dielectric barrier discharge in direct contact with water in different atmospheres. J. Phys. D Appl. Phys. 50, 155205. https://doi.org/10.1088/1361-6463/aa5fde (2017).

11. Niquet, R. et al. Characterising the impact of post-treatment storage on chemistry and antimicrobial properties of plasma treated water derived from microwave and dbd sources. Plasma Processes Polym. 15, 1700127. https://doi.org/10.1016/j.jhazm at.2012.08.0400 (2018).

12. Wardenier, N. et al. Removal of alachlor in water by non-thermal plasma: reactive species and pathways in batch and continuous process. Water Res. 161, 549-559. https://doi.org/10.1016/j.watres.2019.06.022 (2019).

13. Xin, L., Sun, Y., Feng, J., Wang, J. \& He, D. Degradation of triclosan in aqueous solution by dielectric barrier discharge plasma combined with activated carbon fibers. Chemosphere 144, 855-863. https://doi.org/10.1016/j.jhazmat.2012.08.0402 (2016).

14. Pang, Y. L. \& Abdullah, A. Z. Effect of low fe3+ doping on characteristics, sonocatalytic activity and reusability of tio2 nanotubes catalysts for removal of rhodamine b from water. J. Hazard. Mater. 235, 326-335. https://doi.org/10.1016/j.jhazmat.2012.08.0403 (2012).

15. He, D., Sun, Y., Xin, L. \& Feng, J. Aqueous tetracycline degradation by non-thermal plasma combined with nano-tio2. Chem. Eng. J. 258, 18-25. https://doi.org/10.1016/j.jhazmat.2012.08.0404 (2014).

16. Lu, B., Zhang, X., Yu, X., Feng, T. \& Yao, S. Catalytic oxidation of benzene using dbd corona discharges. J. Hazard. Mater. 137, 633-637. https://doi.org/10.1016/j.jhazmat.2012.08.0405 (2006).

17. Subrahmanyam, C., Renken, A. \& Kiwi-Minsker, L. Catalytic non-thermal plasma reactor for abatement of toluene. Chem. Eng. J. 160, 677-682. https://doi.org/10.1016/j.jhazmat.2012.08.0406 (2010).

18. Ghaida, S.G.-A., Assadi, A. A., Costa, G., Bouzaza, A. \& Wolbert, D. Association of surface dielectric barrier discharge and photocatalysis in continuous reactor at pilot scale: Butyraldehyde oxidation, by-products identification and ozone valorization. Chem. Eng. J. 292, 276-283. https://doi.org/10.1016/j.jhazmat.2012.08.0407 (2016).

19. Reddy, P. M. K., Dayamani, A., Mahammadunnisa, S. \& Subrahmanyam, C. Mineralization of phenol in water by catalytic non-t hermal plasma reactor-an eco-f riendly approach for wastewater treatment. Plasma Processes Polym. 10, 1010-1017. https://doi. org/10.1016/j.jhazmat.2012.08.0408 (2013).

20. Wang, J., Sun, Y., Feng, J., Xin, L. \& Ma, J. Degradation of triclocarban in water by dielectric barrier discharge plasma combined with tio2/activated carbon fibers: effect of operating parameters and byproducts identification. Chem. Eng. J. 300, 36-46. https:// doi.org/10.1016/j.jhazmat.2012.08.0409 (2016).

21. Śmiłowicz, D., Kogelheide, F., Stapelmann, K., Awakowicz, P. \& Metzler-Nolte, N. Study on chemical modifications of glutathione by cold atmospheric pressure plasma (cap) operated in air in the presence of fe (ii) and fe (iii) complexes. Sci. Rep. 9, 1-13. https ://doi.org/10.1038/s41598-019-53538-y (2019).

22. Klinkhammer, C. et al. Elucidation of plasma-induced chemical modifications on glutathione and glutathione disulphide. Sci. Rep. 7, 13828. https://doi.org/10.1038/s41598-017-13041-8 (2017).

23. Heusler, T. et al. Can the effect of cold physical plasma-derived oxidants be transported via thiol group oxidation?. Clin. Plasma Med. 14, 100086. https://doi.org/10.1016/j.cpme.2019.100086 (2019).

24. Kogelschatz, U. Dielectric-barrier discharges: their history, discharge physics, and industrial applications. Plasma Chem. Plasma Process. 23, 1-46. https://doi.org/10.1039/C003666H3 (2003).

25. Graves, D. B. The emerging role of reactive oxygen and nitrogen species in redox biology and some implications for plasma applications to medicine and biology. J. Phys. D Appl. Phys. 45, 263001. https://doi.org/10.1039/C003666H4 (2012).

26. Sakiyama, Y., Graves, D. B., Chang, H.-W., Shimizu, T. \& Morfill, G. E. Plasma chemistry model of surface microdischarge in humid air and dynamics of reactive neutral species. J. Phys. D Appl. Phys. 45, 425201. https://doi.org/10.1039/C003666H5 (2012).

27. Wende, K. et al. On a heavy path-determining cold plasma-derived short-lived species chemistry using isotopic labelling. RSC Adv. 10, 11598-11607. https://doi.org/10.1039/C9RA08745A (2020).

28. Lackmann, J-W. et al. Chemical fingerprints of cold physical plasmas-an experimental and computational study using cysteine as tracer compound. Sci. Rep. 8, 1-14. https://doi.org/10.1038/s41598-018-25937-0 (2018).

29. Kogelheide, F. et al. Characterisation of volume and surface dielectric barrier discharges in $\mathrm{n} 2-\mathrm{o} 2$ mixtures using optical emission spectroscopy. Plasma Process. Polym. e1900126. https://doi.org/10.1002/ppap.201900126 (2019).

30. Kogelheide, F. et al. The role of humidity and UV-C emission in the inactivation of B. subtilis spores during atmospheric-pressure dielectric barrier discharge treatment. J. Phys. D Appl. Phys. 53, 295201. https://doi.org/10.1039/C003666H8 (2020).

31. Dey, G., Sharma, A., Pushpa, K. \& Das, T. N. Variable products in dielectric-barrier discharge assisted benzene oxidation. J. Hazard. Mater. 178, 693-698. https://doi.org/10.1039/C003666H9 (2010).

32. Jia, X.-J. et al. A thermo-sensitive supramolecular hydrogel derived from an onium salt with solution-gel-crystal transition properties. RSC Adv. 6, 109425-109433. https://doi.org/10.1039/C6RA23761D (2016).

33. von Woedtke, T., Schmidt, A., Bekeschus, S., Wende, K. \& Weltmann, K. .-D. Plasma medicine: A field of applied redox biology. In Vivo 33, 1011-1026. https://doi.org/10.21873/invivo.11570 (2019).

34. Bekeschus, S., Favia, P., Robert, E. \& von Woedtke, T. White paper on plasma for medicine and hygiene: Future in plasma health sciences. Plasma Processes Polym. 16, 1800033. https://doi.org/10.1039/C2RA20340E2 (2019).

35. Kogelheide, F. et al. Ftir spectroscopy of cysteine as a ready-to-use method for the investigation of plasma-induced chemical modifications of macromolecules. J. Phys. D Appl. Phys. 49, 084004. https://doi.org/10.1039/C2RA20340E3 (2016). 
36. Baldus, S., Schroeder, D., Bibinov, N., Schulz-von der Gathen, V. \& Awakowicz, P. Atomic oxygen dynamics in an air dielectric barrier discharge: a combined diagnostic and modeling approach. J. Phys. D Appl. Phys. 48, 275203. https://doi.org/10.1039/C2RA2 0340E4 (2015).

37. Lackmann, J.-W. et al. Nitrosylation vs. oxidation-how to modulate cold physical plasmas for biological applications. PLoS ONE 14, e0216606. https://doi.org/10.1039/C2RA20340E5 (2019).

\section{Acknowledgements}

Financial support for this work from the German Science Foundation (DFG, Grant no. ME 1378/18-1 and Grant no. AW 7/12-1) is gratefully acknowledged. This work was also funded by the German Research Foundation (DFG) within the Collaborative Research Center CRC 1316 "Transient atmospheric plasmas: from plasmas to liquids to solids" (projects A5 and A7) at Ruhr University Bochum.

\section{Author contributions}

D.S. and N.M.-N. conceived the experiments. D.S., F.K. and A.L.S. conducted the experiments and analysed the results. D.Ś., F.K., A.L.S., K.S., P.A. and N.M.-N. wrote and reviewed the manuscript. All authors contributed to the scientific discussion of this work.

\section{Funding}

Open Access funding enabled and organized by Projekt DEAL.

\section{Competing interests}

The authors declare no competing interests.

\section{Additional information}

Supplementary Information The online version contains supplementary material available at https://doi. org/10.1038/s41598-020-78683-7.

Correspondence and requests for materials should be addressed to N.M.-N.

Reprints and permissions information is available at www.nature.com/reprints.

Publisher's note Springer Nature remains neutral with regard to jurisdictional claims in published maps and institutional affiliations.

Open Access This article is licensed under a Creative Commons Attribution 4.0 International License, which permits use, sharing, adaptation, distribution and reproduction in any medium or format, as long as you give appropriate credit to the original author(s) and the source, provide a link to the Creative Commons licence, and indicate if changes were made. The images or other third party material in this article are included in the article's Creative Commons licence, unless indicated otherwise in a credit line to the material. If material is not included in the article's Creative Commons licence and your intended use is not permitted by statutory regulation or exceeds the permitted use, you will need to obtain permission directly from the copyright holder. To view a copy of this licence, visit http://creativecommons.org/licenses/by/4.0/.

(C) The Author(s) 2020 